\title{
On the detections of $\mathrm{C}_{60}$ and derivatives in circumstellar environments
}

\author{
Yong Zhang and Sun Kwok \\ Department of Physics, University of Hong Kong, Pokfulam, Hong Kong, China
}

(Received October 31, 2012; Revised May 20, 2013; Accepted June 18, 2013; Online published October 24, 2013)

\begin{abstract}
$\mathrm{C}_{60}$ (buckminsterfullerene) was recently discovered in a variety of circumstellar environments by the Spitzer Space Telescope, suggesting that the envelopes around evolved stars are active sites for the synthesis of fullerenes. However, the physical state, excitation mechanism, and formation route of circumstellar $\mathrm{C}_{60}$ are not completely understood so far. These open issues are discussed in this paper. For that purpose we investigate the observed wavelengths and strengths of $\mathrm{C}_{60}$ bands and compare them with the experimental values. We also statistically study the environments and emission properties of the $\mathrm{C}_{60}$ sources. We would like to stress that improved flux measurements and more accurate Einstein coefficients are required to draw solid conclusions. Furthermore, we present possible detections of hydrogenated $\mathrm{C}_{60}$ and $\mathrm{C}_{60}^{+}$, and discuss their implications on fullerene chemistry in circumstellar environments.
\end{abstract}

Key words: Infrared, ISM, AGB and post-AGB, circumstellar matter, molecules.

\section{Introduction}

The $I_{\mathrm{h}}$-symmetrical buckminsterfullerene $\mathrm{C}_{60}$, arranged as 12 pentagons and 20 hexagons, is the smallest closed carbon cage molecule (fullerene) satisfying the isolated pentagon rule (IPR; followed by higher fullerenes $\mathrm{C}_{70}, \mathrm{C}_{74}$, $\mathrm{C}_{76}, \mathrm{C}_{78}, \mathrm{C}_{80}$, and so on), and thus is remarkably stable. Although its existence has been early predicted (Osawa, 1970; Bochvar and Galpern, 1973), $\mathrm{C}_{60}$ was first discovered in laboratory experiments simulating the circumstellar chemistry (Kroto et al., 1985). Krätschmer et al. (1990b) developed a method to efficiently produce $\mathrm{C}_{60}$ in the laboratory, making it possible to study in detail its electronic and vibrational properties. Because of its high stability and symmetry, $\mathrm{C}_{60}$ can be taken as a benchmark to study other fullerenes. $\mathrm{C}_{60}$ and its derivatives have long been suspected to be present in the universe. The search for this compound in nature started soon after its synthesis in the laboratory, and its detections in geological materials and meteorites have been reported by several groups (e.g. Buseck et al., 1992; Becker and Bada, 1994; Becker et al., 1994; Heymann et al., 1996) ${ }^{1}$.

The detection of $\mathrm{C}_{60}$ in deep space has proven a long lasting challenging task (Snow and Seab, 1989; Clayton et al., 1995; Kwok et al., 1999; Moutou et al., 1999; Herbig, 2000; Sassara et al., 2001). $\mathrm{C}_{60}$ has three broad peaks at 216,264 , and $339 \mathrm{~nm}$ in its electronic spectrum, and four infrared (IR) vibrational modes at 7.0, 8.5, 17.4, and $18.9 \mu \mathrm{m}$. Hydrogen-poor and carbon-rich circumstellar envelopes, such as R Coronae Borealis (RCB) stars (Goeres and Sedlmayr, 1992), are analogous to the laboratory conditions for the synthesis of fullerenes and thus were sug-

Copyright (c) The Society of Geomagnetism and Earth, Planetary and Space Sciences (SGEPSS); The Seismological Society of Japan; The Volcanological Society of Japan; The Geodetic Society of Japan; The Japanese Society for Planetary Sciences; TERRAPUB

doi:10.5047/eps.2013.06.003 gested to be ideal sites to search for $\mathrm{C}_{60}$; however, this was not supported by observations (García-Hernández et al., 2011b). So far, there is no successful report on the detection of electronic transitions of $\mathrm{C}_{60}$. The Infrared Spectrograph (IRS; Houck et al., 2004) on the Spitzer Space Telescope (Spitzer; Werner et al., 2004) now provides an unprecedented opportunity to detect the IR vibrational transitions. The first convincing detection was recently made by Cami et al. (2010), who detected $\mathrm{C}_{60}$ and $\mathrm{C}_{70}$ in the Spitzer/IRS spectrum of the young planetary nebula (PN) Tc 1. Soon after, $\mathrm{C}_{60}$ was detected in a variety of evolved stars, including PNs in the Milky Way and the Magellanic Clouds, a protoplanetary nebula (PPN), post asymptotic giant branch (AGB) stars, modestly hydrogen-deficient RCB stars, and a peculiar binary object (García-Hernández et al., 2010, 2011a, b; Clayton et al., 2011; Gielen et al., 2011; Zhang and Kwok, 2011; Evans et al., 2012; Roberts et al., 2012). Fullerenes may also be widely present in the interstellar medium (ISM). $\mathrm{C}_{60}^{+}$has been proposed as the possible carrier of diffuse interstellar bands (Léger et al., 1988), and its electronic spectra in $\mathrm{Ar}$ and Ne matrices indeed show a reasonable match to two near-IR diffuse bands (Foing and Ehrenfreund, 1994). Photoabsorption by fullerenes and multi-layered fullerenes (buckyonions) has been suggested as the origin of the $217 \mathrm{~nm}$ extinction feature (de Heer and Ugarte, 1993; Iglesias-Groth, 2004; Li et al., 2008). Recently, the $\mathrm{C}_{60}$ IR bands have been detected in reflection nebulae (RNs) and the Orion nebula (Sellgren et al., 2010; Rubin et al., 2011). Furthermore, Roberts et al. (2012) detected $\mathrm{C}_{60}$ in pre-main-sequence objects including young stellar objects and a Herbig Ae/Be star. These detections suggest that $\mathrm{C}_{60}$ can be formed in a short time scale between AGB and PN stages, and can survive (or be resynthesized under favorable conditions) in the ISM.

\footnotetext{
${ }^{1}$ However, some contrary results have been reported by different research groups (e.g. de Vries et al., 1993; Ebbesen et al., 1995; Heymann, 1997).
} 
However, based on a laboratory spectroscopic investigation, Duley and $\mathrm{Hu}$ (2012) presented that the 7.0, 8.5, 17.4, and $18.9 \mu \mathrm{m}$ features in the spectra dominated by aromatic infrared bands (AIBs) actually arise from proto-fullerenes that are precursors of $\mathrm{C}_{60}$, and thus the $\mathrm{C}_{60}$ molecules were detected only in sources that do not show AIBs. GarcíaHernández et al. (2012b) did not detect $\mathrm{C}_{60}$ electronic transitions in the optical spectrum of a RCB star that exhibit AIBs and the (supposed) $\mathrm{C}_{60}$ IR features, and suggested that this supported the conclusion of Duley and $\mathrm{Hu}$ (2012). However, it is surprising that $\mathrm{C}_{60}$ electronic transitions are not detected in the high-quality optical spectrum of the strongest $\mathrm{C}_{60}$ source without AIB emission, Tc 1 (GarcíaHernández and Díaz-Luis, 2013).

The formation route of $\mathrm{C}_{60}$ is a subject of debate. Investigating this problem may provide significant insights into the circumstellar chemistry. The experiments of Kroto et al. (1985) suggest that small carbon clusters can selfassemble into $\mathrm{C}_{60}$ in a hydrogen-poor environment (bottomup), and otherwise the formation of polycyclic aromatic hydrocarbons (PAHs) is favored. This scenario is supported by the fact that no AIBs are detected in the PN Tc 1 (Cami et al., 2010). Nevertheless, Jäger et al. (2009) found that fullerenes can be formed by gas-phase condensation reactions in the presence of hydrogen at very high temperature $(>3500 \mathrm{~K})$. Another formation mechanism of $\mathrm{C}_{60}$ is through shock- or UV-induced decomposition of hydrogenated amorphous carbon (HAC) grains (topdown), through which PAHs can be simultaneously formed (García-Hernández et al., 2010; Bernard-Salas et al., 2012; Micelotta et al., 2012). The supporting evidence comes from the facts that most of the $\mathrm{C}_{60}$ sources also exhibit AIBs, and $\mathrm{C}_{60}$ is not detected in extremely hydrogen-poor RCB stars (García-Hernández et al., 2011b). A similar mechanism was proposed by Berné and Tielens (2012), in which $\mathrm{C}_{60}$ is formed through $\mathrm{UV}$-induced dehydrogenation and isomerization of graphenes. This model can explain the observation of the RN NGC 7023 by Sellgren et al. (2010) that the $\mathrm{C}_{60}$ /AIB flux ratios decrease with increasing distance from the central star. Moreover, Boersma et al. (2012) found that $\mathrm{C}_{60}$ can coexist with the carriers of $\mathrm{AIBs}$ in the shielded regions of Orion.

A related question is what drives the excitation of $\mathrm{C}_{60}$ IR emission. Thermal excitation of solid fullerenes (Cami et al., 2010; García-Hernández et al., 2011a) and UVexcitation of gas-phase $\mathrm{C}_{60}$ molecules (Sellgren et al., 2010) have been discussed. The first systematical comparison of the two mechanisms was made by Bernard-Salas et al. (2012) who found that the IR spectra of three PNs with strong $\mathrm{C}_{60}$ features favor fluorescence as the excitation mechanism. However, Roberts et al. (2012) and GarcíaHernández et al. (2012a) argued that thermal excitation can better explain the observed $\mathrm{C}_{60}$ flux ratios.

Mapping the spatial distributions of $\mathrm{C}_{60}$ and AIBs is essential to understand the formation routes of fullerenes in astrophysics. However, this is very difficult for circumstellar envelopes given their compactness and the weakness of $\mathrm{C}_{60}$ emission. Another approach to investigate this problem is to probe hydrides of fullerenes (fulleranes). The presence of fulleranes in space has been predicted (e.g. Web- ster, 1992, 1993; Petrie and Bohme, 2000; Cataldo and Iglesias-Groth, 2009). When hydrogen atoms are attached to carbon atoms of $\mathrm{C}_{60}$, the conversion of delocalized $\pi$ into $\sigma$-character orbitals decreases the bond angle strain. Therefore, when exposed to atomic hydrogen, $\mathrm{C}_{60}$ can be quickly hydrogenated into $\mathrm{C}_{60} \mathrm{H}_{m}(m=2,4, \ldots, 60)$. On the other hand, heavily hydrogenated $\mathrm{C}_{60}$ is highly unstable because of large strain resulting from hydrogen-hydrogen repulsion. It follows that moderately hydrogenated $\mathrm{C}_{60}$ is likely to exist in circumstellar environments. Indeed, in laboratory conditions, it is easy to produce $\mathrm{C}_{60} \mathrm{H}_{36}$ that can further form $\mathrm{C}_{60} \mathrm{H}_{18}$ through thermal annealing (e.g. IglesiasGroth et al., 2012) but the production of fulleranes with a larger or lower degree of hydrogenation is much more difficult. Roberts et al. (2012) noticed that two $\mathrm{C}_{60}$ sources also exhibit the very rare $\mathrm{C}-\mathrm{H}$ emission from hydrogen-coated nanodiamonds, a species that can be formed together with fullerenes, so it is reasonable to believe that $\mathrm{C}-\mathrm{H}$ emission from $\mathrm{C}_{60} \mathrm{H}_{m}$ is also detectable in some $\mathrm{C}_{60}$ sources.

In this paper, we study the properties of all the reported $\mathrm{C}_{60}$ sources and compare the observations with existing experimental and theoretical results. In Section 2, we investigate the wavelengths and widths of the $C_{60}$ IR bands, aiming at understanding the physical state of circumstellar $\mathrm{C}_{60}$. In Sections 3 and 4, we respectively discuss the excitation and formation problems of fullerenes based on the measured band strengths. In Section 5, we present a search for fulleranes in the carbon-rich PPN IRAS 01005+7910 utilizing the spectrum obtained by the Infrared Space Observatory (ISO; Kessler et al., 1996), and briefly discuss $\mathrm{C}_{60}^{+}$and $\mathrm{C}_{60}^{-}$in the PN Tc 1. The conclusions are given in Section 6.

\section{Wavelengths of the IR-active Bands}

$\mathrm{C}_{60}$ has 174 normal vibrational modes that are distributed among 46 frequencies owning to its icosahedral symmetry. Of these modes, only 4 IR-active vibrational bands of $\mathrm{F}_{1 u}$ symmetry and 10 Raman-active bands of $\mathrm{A}_{g}$ and $\mathrm{H}_{g}$ symmetries have been observed in laboratory experiments. Throughout this paper we only focus on the four IR $\mathrm{C}_{60}$ bands that are of astrophysical interest. Because of intermolecular interactions, the wavelengths of IR bands from solid $\mathrm{C}_{60}$ clusters might differ from those from gas-phase molecules and depend on the environment temperatures. Therefore, the comparison between the observed and experimental wavelengths of the $\mathrm{C}_{60}$ IR bands enables us to investigate the physical state of circumstellar fullerenes.

Krätschmer et al. (1990a) and Frum et al. (1991) have made the laboratory measurements of the solid- and gasphase spectra of $\mathrm{C}_{60}$, respectively. By comparing their results and the observed spectra, Evans et al. (2012) concluded that $\mathrm{C}_{60}$ in the peculiar binary $\mathrm{XX}$ Oph is in the solid phase. Temperature-dependent studies of $\mathrm{C}_{60}$ IR spectra have been presented by Chase et al. (1992), Nemes et al. (1994), and Iglesias-Groth et al. (2011) showing that the peak wavelengths shift to higher values with increasing temperature. In Fig. 1, we compare these experimental wavelengths and the spectra of three $\mathrm{C}_{60}$ sources, Tc 1 , NGC 7023, and IRAS 01005+7910. There is no evidence showing that the peak wavelengths of $\mathrm{C}_{60}$ features shift between different sources ${ }^{2}$, suggesting that fullerenes in all 

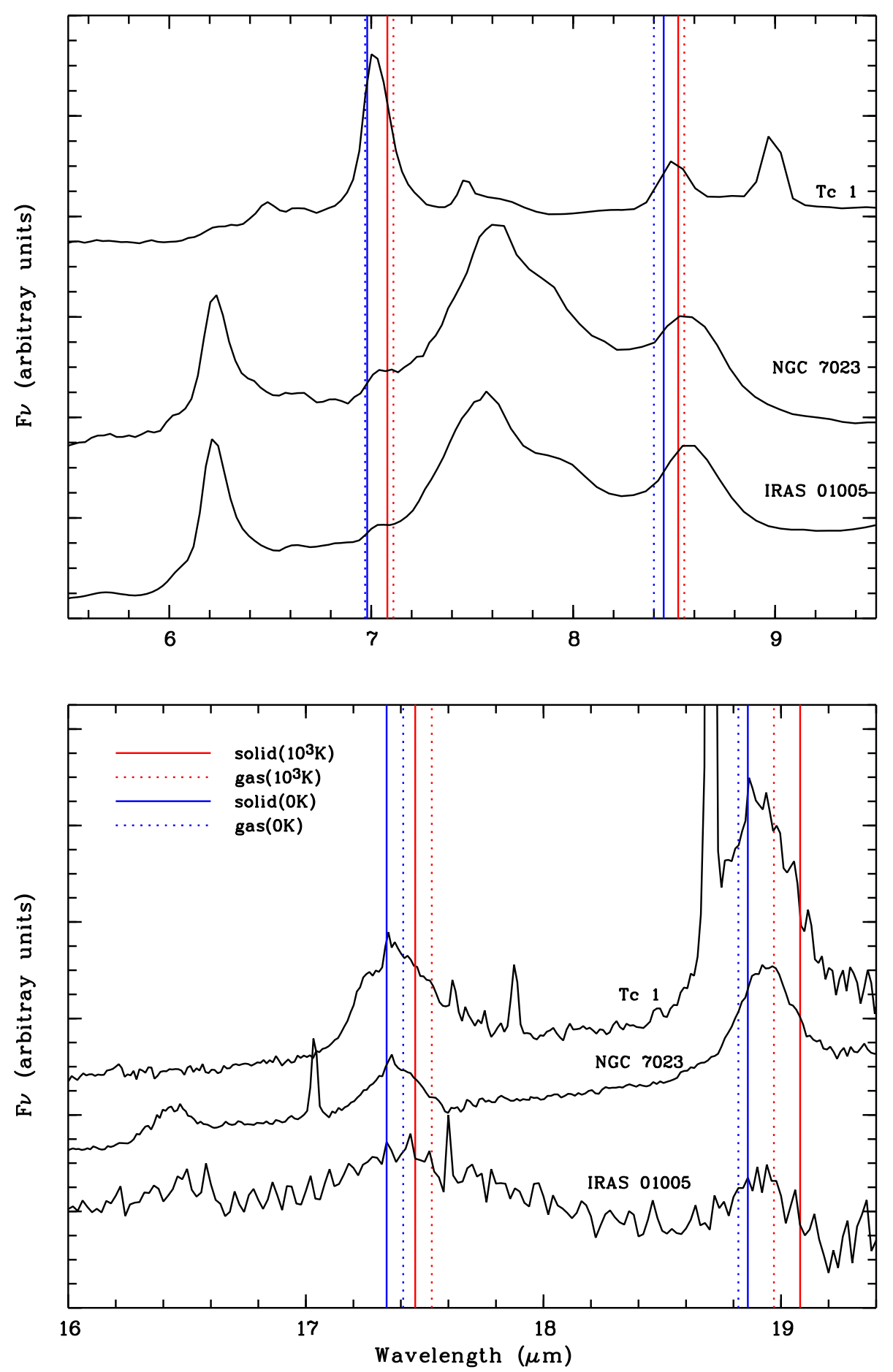

Fig. 1. The comparison of the experimental wavelengths of the four IR bands of solid- and gas-phase $\mathrm{C}_{60}$. The solid black curves are the observed spectra of IRAS 01005+7910, NGC 7023, and Tc 1 (courtesy of Kris Sellgren, Jan Cami, and Jeronimo Bernard-Salas). Note that the 7.0, 8.5, and $17.4 \mu \mathrm{m}$ features are blended with AIBs in the spectra of NGC 7023 and IRAS $01005+7910$.

the circumstellar envelopes are probably in a similar physical state. An inspection of Fig. 1 indicates that the peak wavelengths of the $17.4 \mu \mathrm{m}$ feature seem to suggest that circumstellar $\mathrm{C}_{60}$ is in the solid phase at a low temperature $(<500 \mathrm{~K})$ rather than in the gas phase. The conclusion, however, is arguable in that the spectra of NGC 7023 and IRAS $01005+7910$ exhibit the $16.4 \mu \mathrm{m}$ features, and thus the $17.4 \mu \mathrm{m}$ feature is probably blended with an AIB (Sellgren et al., 2010; Berné and Tielens, 2012). The observed peak-wavelengths of the other three $\mathrm{C}_{60}$ features lie well in- side the experimental wavelength ranges of both solid- and gas-phase $\mathrm{C}_{60}$. Therefore, we cannot completely rule out a gas-phase origin although a solid-phase origin is favored.

Experimentally, the 17.4 and $18.9 \mu \mathrm{m}$ features in the

${ }^{2}$ Note that the $8.5 \mu \mathrm{m}$ feature in the spectra of NGC7023 and IRAS $01005+7910$ is essentially invisible because of severe blending with the $\mathrm{AIB}$ at $8.6 \mu \mathrm{m}$, and the $7.0 \mu \mathrm{m}$ feature in Tc 1 is contaminated with a [Ar II] line at $7.0 \mu \mathrm{m}$. This statement also applies to most of the $\mathrm{C}_{60}$ sources, and thus additional complexity can be introduced into the discussion of band strength ratios in the next section. 


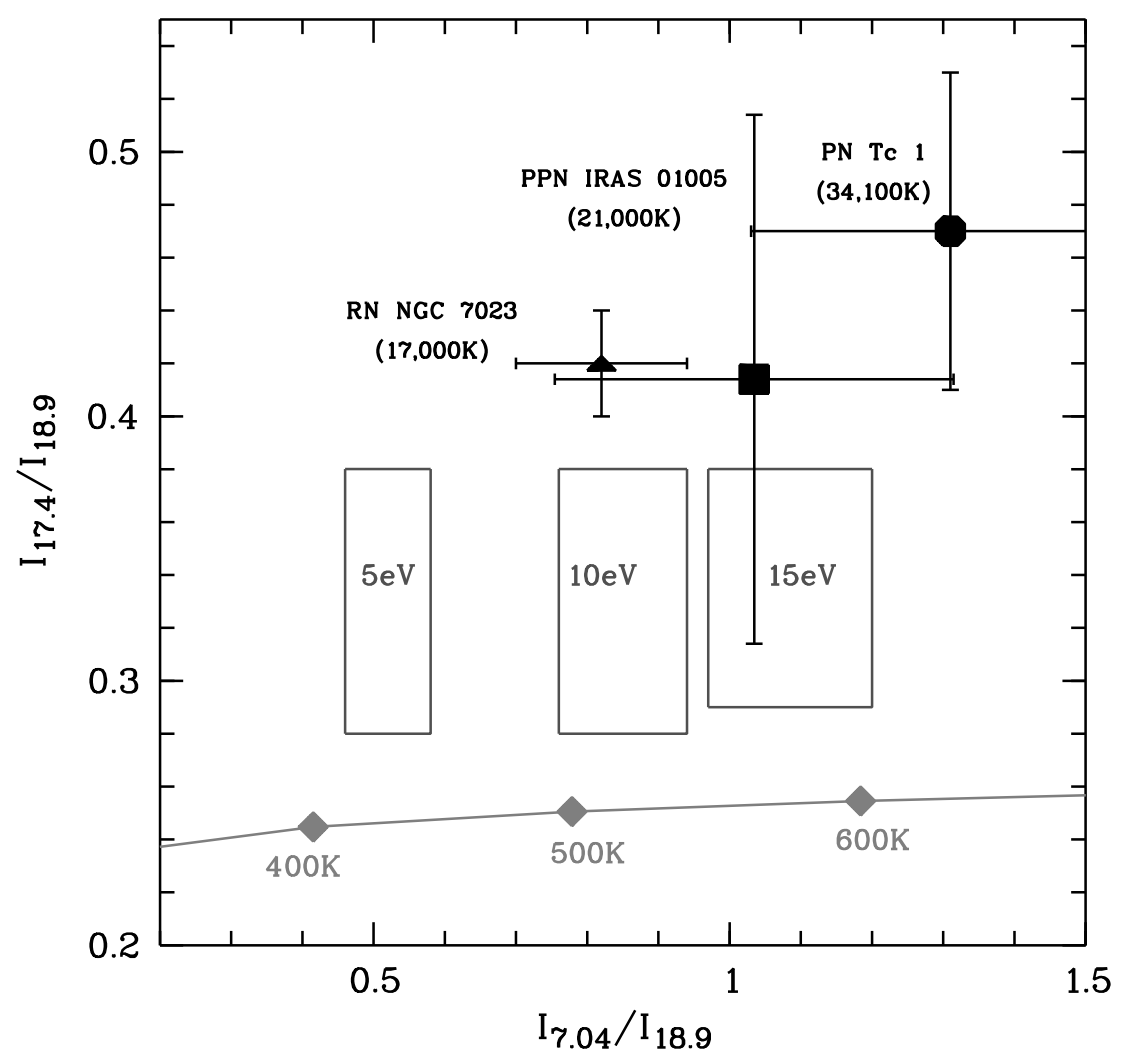

Fig. 2. The $17.4 \mu \mathrm{m} / 18.9 \mu \mathrm{m}$ versus $7.0 \mu \mathrm{m} / 18.9 \mu \mathrm{m}$ band ratios for NGC 7023, IRAS 01005+7910, and Tc 1 . The effective temperatures of their excitation sources are given in brackets. The closed boxes represent the predictions of UV-excitation model by Sellgren et al. (2010). The average photon-energy is given within each box. The curve marked with lozenges represents the predictions of thermal model. The A-values for both models are taken from the same literature (Choi et al., 2000).

gas-phase IR spectrum have a full-width at half maximum (FWHM) of about $0.4 \mu \mathrm{m}$ at $1065 \mathrm{~K}$ (Frum et al., 1991). Chase et al. (1992) found that the band widths of $\mathrm{C}_{60}$ shift little in the solution and in the solid state, suggesting that $\mathrm{C}_{60}$ in the solid state is virtually a gas-phase-like, freely rotating molecule. The band widths are partially due to vibration-rotation coupling, scaling roughly with the square root of the temperature. Therefore, one can, in principle, estimate the temperature by comparing the observed band widths with the experimental values. A comparison between the experimental results of Frum et al. (1991) and Chase et al. (1992) suggests that the band widths of solidphase $\mathrm{C}_{60}$ are 2-3 times narrower than those of gas-phase at the same temperature. The FWHMs of the observed 17.4 and $18.9 \mu \mathrm{m}$ features are $0.26-0.36 \mu \mathrm{m}$, implying a gas temperature of 400-900 K. The implicated temperature might be higher if $\mathrm{C}_{60}$ is in the solid phase. While interesting, these results should be viewed with some caution because they might be related to the experimental conditions, which are quite different from those of circumstellar envelopes. The 17.4 and $18.9 \mu \mathrm{m}$ features might be blended with weak $\mathrm{C}_{70}$ emission (see, e.g., figure 1 of Cami et al., 2010), slightly affecting their FWHMs. Moreover, if the $\mathrm{C}_{60}$ bands arise from regions with large temperature fluctuations, the situation will be complicated in that the shifts of peak wavelengths can make an additional contribution to the band broadening.

\section{On the Excitation of $\mathrm{C}_{60}$ Vibrational Spectra}

It is essential to understand the excitation mechanism of fullerene emission as it enables us to accurately determine the abundance of $\mathrm{C}_{60}$ and establish how useful the $\mathrm{C}_{60}$ bands are to probe circumstellar environments. Two possible scenarios have been discussed in previous paper, but results remain conflicting. Cami et al. (2010) found that a thermal model can interpret the $\mathrm{C}_{60}$ emission in $\mathrm{Tc} 1$. In that case, $\mathrm{C}_{60}$ is attached to dust grains which are in equilibrium with the UV radiation field, and one can calculate the thermal temperature from the Boltzmann excitation diagram of $\mathrm{C}_{60}$ band ratios. García-Hernández et al. (2011a) derived thermal temperatures of $200-500 \mathrm{~K}$ for a sample of $\mathrm{C}_{60^{-}}$ detected PNs. On the other hand, Sellgren et al. (2010) assumed that $\mathrm{C}_{60}$ is isolated free molecules in the gas phase and has a similar excitation mechanism with PAHs as discussed by Allamandola et al. (1989). In this scenario, $\mathrm{C}_{60}$ is excited to high electronic states by absorbing UV starlight, and then the electronic energy is quickly redistributed via internal conversion, followed by IR emission through cascading down the vibrational ladder to the ground vibrational state. Since the probabilities of UV-photon absorption and IR-photon emission respectively depend on the absorption cross-sections and Einstein A-values of the corresponding energy levels, a Monte Carlo technique ${ }^{3}$ can be used to simulate this process. Therefore, if the UV-excitation model is valid, the $\mathrm{C}_{60}$ IR bands can be used to probe the circumstellar radiation field. The model of Sellgren et al. (2010) can reasonably explain the $\mathrm{C}_{60}$ intensity ratios ob- 
Table 1. $\mathrm{C}_{60}$ band strengths normalized to that of the $18.9 \mu \mathrm{m}$ feature.

\begin{tabular}{lcccc}
\hline Ref. & $7.0 \mu \mathrm{m}$ & $8.5 \mu \mathrm{m}$ & $17.4 \mu \mathrm{m}$ & $18.9 \mu \mathrm{m}$ \\
\hline \multicolumn{2}{c}{ Experiments } & & \\
\hline Chase et al. (1992) & 0.34 & 0.28 & 0.34 & 1.0 \\
Fu et al. (1992) & 0.36 & 0.29 & 0.39 & 1.0 \\
Martin et al. (1993) & 0.38 & 0.45 & 0.48 & 1.0 \\
Winkler et al. (1994) & 0.36 & 0.16 & 0.28 & 1.0 \\
Onoe and Takeuchi (1996) & 0.24 & 0.22 & 0.30 & 1.0 \\
Hara et al. (1997) & 0.38 & 0.20 & 0.27 & 1.0 \\
Choi et al. (2000) & 0.46 & 0.31 & 0.26 & 1.0 \\
Iglesias-Groth (2004) & 0.27 & 0.26 & 0.43 & 1.0 \\
\hline & Theoretical calculations & & \\
\hline Bertsch et al. (1995) & 2.7 & 0.17 & 0.47 & 1.0 \\
Esfarjani et al. (1998) & 1.7 & 0.18 & 0.08 & 1.0 \\
Stanton and Newton (1988) & 4.4 & 1.9 & 0.33 & 1.0 \\
Adams et al. (1994) & 0.31 & 1.4 & 0.65 & 1.0 \\
Bertsch et al. (1995) & 0.41 & 0.59 & 0.65 & 1.0 \\
Giannozzi and Baroni (1994) & 0.57 & 0.36 & 0.63 & 1.0 \\
\hline
\end{tabular}

served in NGC 7023, but predicts too large $I_{7.0} / I_{18.9}$ ratios in NGC 2023. A recent study of three PNs by Bernard-Salas et al. (2012) does not favor the thermal model because (1) the excitation classes of these PNs are very different while the $\mathrm{C}_{60}$ flux ratios are fairly similar, and (2) $\mathrm{C}_{60}$ is located in a region far from the central star and would be difficult to be heated to the deduced thermal temperatures. However, a different conclusion was subsequently drawn by GarcíaHernández et al. (2012a) who subtracted the contribution of $\mathrm{C}_{70}$ to the observed $7.0 \mu \mathrm{m}$ flux and found that the UVexcitation model cannot explain the $\mathrm{C}_{60}$ flux ratios for a larger sample of PNs. Bernard-Salas et al. (2012) did not take into account the contribution of $\mathrm{C}_{70}$ because the band strengths cannot be accurately estimated without appropriate excitation model.

To investigate this problem, in Fig. 2 we compare the $17.4 \mu \mathrm{m} / 18.9 \mu \mathrm{m}$ and $7.0 \mu \mathrm{m} / 18.9 \mu \mathrm{m}$ band ratios for three well $\mathrm{C}_{60}$-detected sources at different evolutionary stages. The predictions of UV-excitation (Sellgren et al., 2010) and thermal models are also plotted in Fig. 2, where the A-values are derived from Choi et al. (2000) for both models. The thermal model is constructed using the same way as described by Bernard-Salas et al. (2012). Similar to the thermal model for $>500 \mathrm{~K}$, the UV-excitation model predicts a nearly invariable $17.4 \mu \mathrm{m} / 18.9 \mu \mathrm{m}$ band ratio (also see Bernard-Salas et al., 2012). An inspection of Fig. 2 shows that the observations are situated at positions closer to the predictions of the UV-excitation model. In the scenario of UV-excitation, the $7.0 \mu \mathrm{m} / 18.9 \mu \mathrm{m}$ band ratios increase with increasing UV radiation. This is consistent with the observational facts that among the three sources the central star of the PN Tc 1 has the highest effective temperature, and it is followed by the PPN IRAS $01005+7910$ and the RN NGC 7023. Nevertheless, the observed $17.4 \mu \mathrm{m}$ / $18.9 \mu \mathrm{m}$ band ratios appear larger than the model predications. This is partially due to the contamination of an

\footnotetext{
${ }^{3}$ The Monte Carlo model of Sellgren et al. (2010) applies to single-photon excitation. If the UV radiation field is sufficiently intense, it is more appropriate to use the statistical equilibrium method (A. Li, private communication).
}

AIB to the $17.4 \mu \mathrm{m}$ feature (except for Tc 1 which does not show AIBs). Another possible cause is that the $A_{17.4}$ value of Choi et al. (2000) has been underestimated. This is also shown in figures 5 and 6 of Bernard-Salas et al. (2012).

In Fig. 3, we compare the A-values obtained from the band strengths given in previous literatures, as listed in Table 1. The theoretical band strengths are remarkably inconsistent with the experimental values. The theoretical calculations, based on semi-empirical or first-principle models, differ significantly in the resultant band strengths, especially for the $7.0 \mu \mathrm{m} / 18.9 \mu \mathrm{m}$ band ratios. The experimental band strengths are more concentrated in Fig. 3 than the theoretical values, yielding an average strength ratio of $(0.35 \pm 0.07):(0.27 \pm 0.09):(0.34 \pm 0.08): 1.0$ for the 7.0, 8.5, 17.4, and $18.9 \mu \mathrm{m}$ bands. We can see that, indeed, the $\mathrm{A}_{17.4}$ value of Choi et al. (2000) is a factor of 1.3 lower than the average value. Through the comparison, we estimate that the A-values have a $20-30 \%$ error. It should be noted that all the experiments were performed in condensed phases and the results might be perturbed by intermolecular effects. Experiments of gas-phase $\mathrm{C}_{60}$ will be invaluable to improve the A-values in the future. In addition, the UV-excitation model also relies on the photoabsorption cross section of $\mathrm{C}_{60}$ which contains some uncertainties (Yasumatsu et al., 1996; Iglesias-Groth et al., 2002; Yagi et al., 2009).

The thermal-model predictions using A-values obtained by different groups are compared in Fig. 4 along with the observed band ratios of all the $\mathrm{C}_{60}$ sources. It is clear that with higher $\mathrm{A}_{17.4}$ value the prediction of thermal models can better match the observed $17.4 \mu \mathrm{m} / 18.9 \mu \mathrm{m}$ flux ratios. Gielen et al. (2011) did not detect the $\mathrm{C}_{60} 7.0 \mu \mathrm{m}$ band in two post-AGB stars. This is understandable in terms of both models since their central stars have relatively low effective temperatures. However, the observed band ratios for most of the $\mathrm{C}_{60}$ sources exhibit a wide variation, and some of them significantly deviate from both model predications. We suspect that the flux measurements of $\mathrm{C}_{60} \mathrm{IR}$ bands, which are subject to the subtraction of continuum emission and the decomposition from other features, prob- 


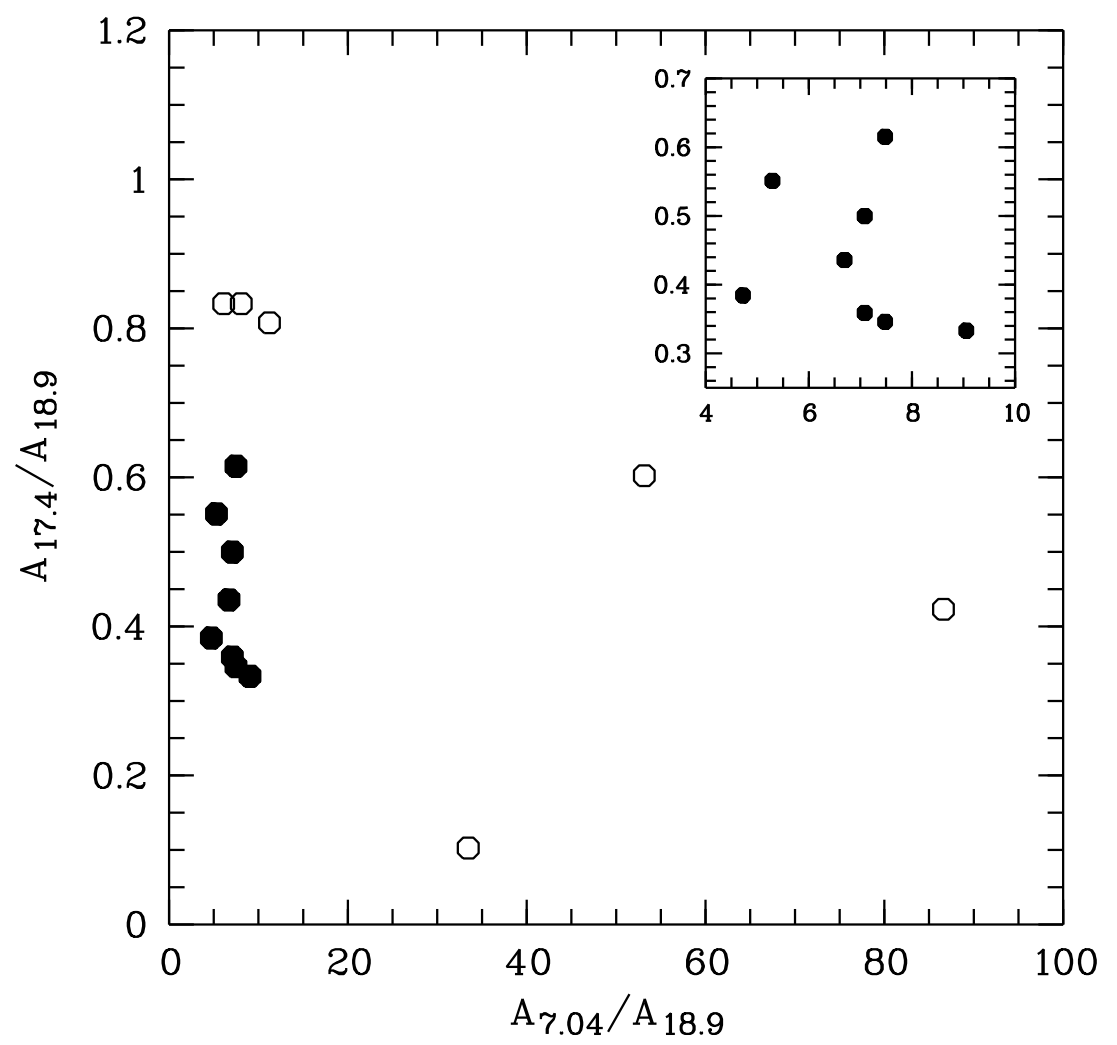

Fig. 3. The comparison of Einstein coefficients (A-values) taken from previous literatures. The open and filled circles represent the theoretical and experimental values, respectively. The insert at the low-left corner is a zoom-in view of the experimental values.

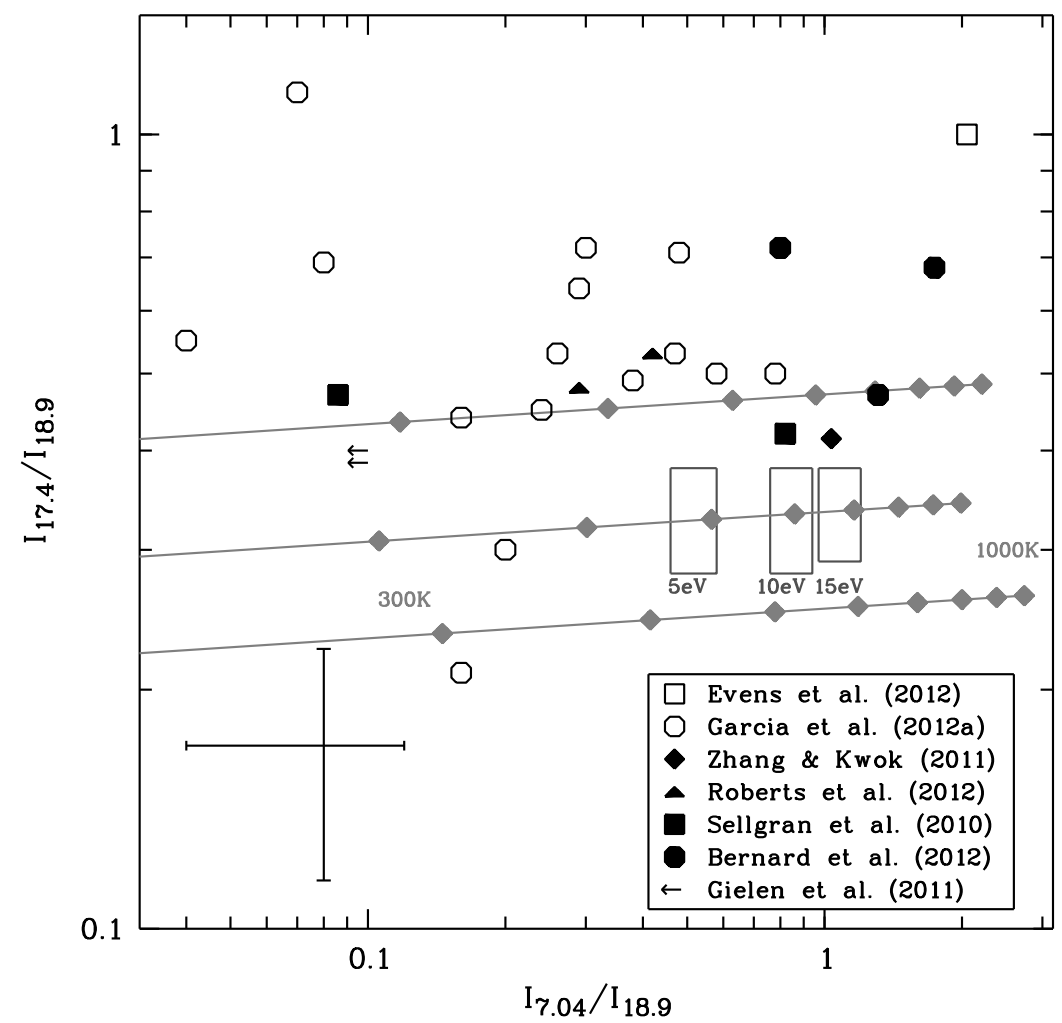

Fig. 4. Same as Fig. 2, but for all the reported $\mathrm{C}_{60}$ sources. The typical error bar is indicated in the lower left-hand corner. The solid curves denote the predicted values by thermal models with temperatures from 300 to $1000 \mathrm{~K}$ at an interval of $100 \mathrm{~K}$, for which the A-values (from up to down) are taken from Martin et al. (1993), Chase et al. (1992), and Choi et al. (2000), respectively. 


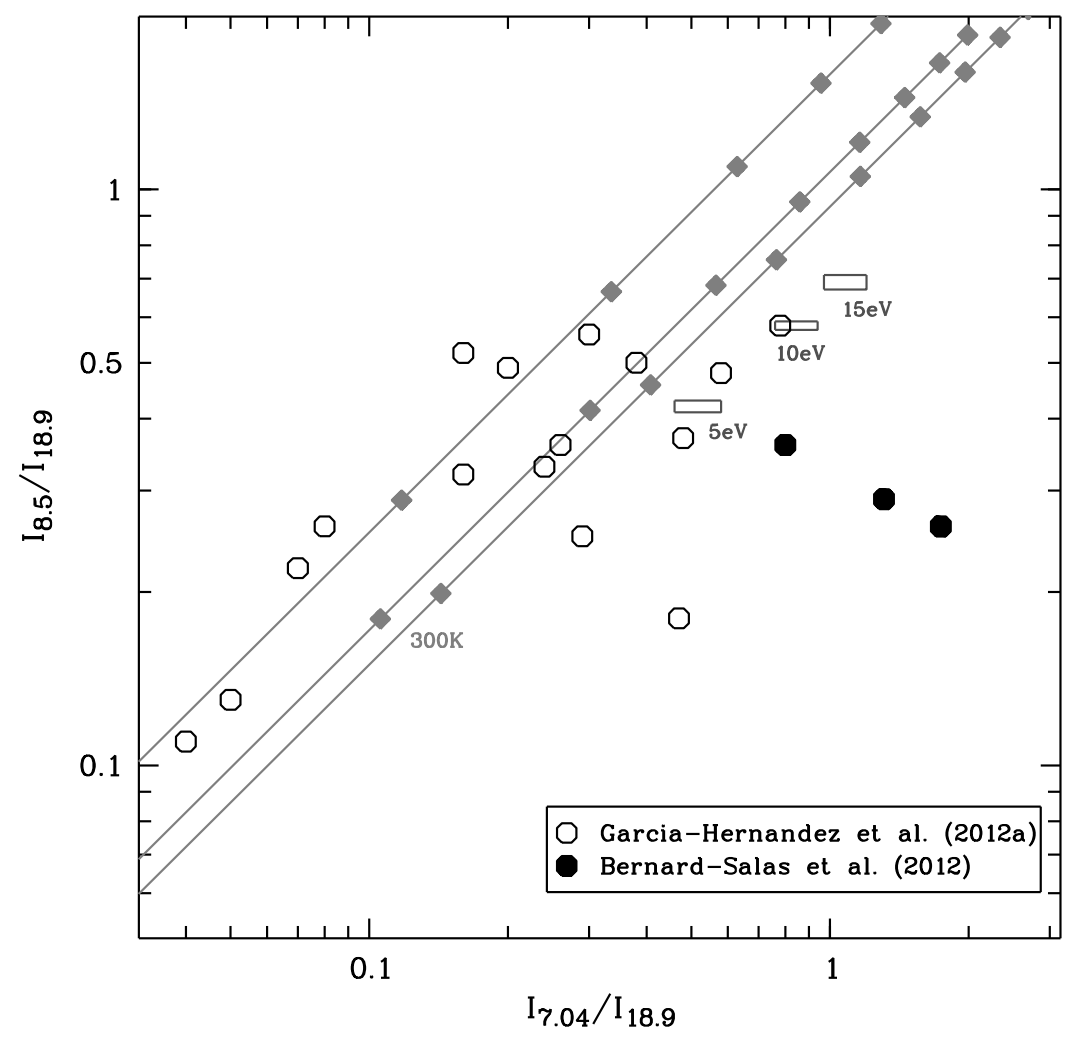

Fig. 5. The $8.5 \mu \mathrm{m} / 18.9 \mu \mathrm{m}$ versus $7.0 \mu \mathrm{m} / 18.9 \mu \mathrm{m}$ band ratios. The symbols are the same as in Fig. 4 . Note that the 7.0 $\mu \mathrm{m}$ band strengths of García-Hernández et al. (2012a) were estimated from thermal models, and thus cannot be used to argue against the fluorescence model (see the text).

ably have rather large uncertainties. For example, even for the strongest fullerene emission PN Tc 1, Bernard-Salas et al. (2012) and García-Hernández et al. (2012a) gave quite different flux ratios.

The $\mathrm{C}_{60} 8.5 \mu \mathrm{m}$ band can be detected only in objects that do not exhibit strong AIBs. In Fig. 5, we compare the 8.5 $\mu \mathrm{m} / 18.9 \mu \mathrm{m}$ and $7.0 \mu \mathrm{m} / 18.9 \mu \mathrm{m}$ band ratios. Compared with those by Bernard-Salas et al. (2012), the band ratios obtained by García-Hernández et al. (2012a) systematically shift toward the left side of the figure. This is attributed to the different estimates for the contamination of $\mathrm{C}_{70}$ emission. Assuming a Boltzmann population ratio, GarcíaHernández et al. (2012a) estimated the strengths of the $\mathrm{C}_{60}$ $7.0 \mu \mathrm{m}$ band from the $8.5,17.4$, and $18.9 \mu \mathrm{m}$ bands. Thus, it is only natural that the data points of García-Hernández et al. (2012a) are more close to the predictions of thermal models, which cannot be used to argue against the conclusion of Bernard-Salas et al. (2012). The main problem of the thermal model is that a high fullerene temperature $($ mostly $>300 \mathrm{~K})$ is required, and hardly agrees with those implicated by dust thermal emission. On the other hand, Fig. 5 demonstrates that the UV-excitation model predicts an unacceptably low average photon energy (mostly $<5$ $\mathrm{eV}$ ). This is the main argument of García-Hernández et al. (2012a) against the UV-excitation model. However, the UV-excitation model of Sellgren et al. (2010) applies only for free-flying $\mathrm{C}_{60}$ molecules. If the circumstellar fullerenes are in a cluster state, more intense radiation field will be expected to excite the $\mathrm{C}_{60}$ IR bands, and the model of Sellgren et al . (2010) only gives the lower limits of the average photon energy. If this was the case, the scattering band ratios in
Figs. 4 and 5 would partially reflect the variation of cluster sizes in these sources.

Some alternative mechanisms, such as the release of chemical energy (Duley and Williams, 2011) and atom impacts (Papoular, 2012), have been introduced to explain the excitation of AIBs. It remains unknown whether these mechanisms play a role on the excitation of $\mathrm{C}_{60}$ IR bands.

\section{On the Origin of $\mathbf{C}_{60}$}

The synthesis of fullerenes in nature has been a subject of intense discussion for many years. It is unclear whether they form through assembly of small carbonbearing molecules (bottom-up) or fragmentations of large compounds (top-down). The discovery of fullerenes in rocks (Buseck et al., 1992) suggests that fullerenes can be generated through a solid-state process. Many interesting scenarios, such as cataclysmic impact, extensive wildfires, chondritic impactor, vaporization of carbon by lightning strike, and pyrolysis of organic matter, have been proposed to explain the formation of fullerenes in geological environments (see Buseck, 2002, and references therein). To investigate $\mathrm{C}_{60}$ formation in circumstellar environments, it is instructive to compare the observations with experimental knowledge. In laboratory, the most effective two ways to produce fullerenes are through vaporization of graphite followed by growth of carbon clusters and through combustion of hydrocarbons. The other experimental routes, such as pyrolysis of hydrocarbons and chemical synthesis, are unlikely to happen under circumstellar environments. The method of graphite evaporation (bottom-up) requires a carbon-rich environment, otherwise the fullerene formation 
is strongly suppressed. However, $\mathrm{C}_{60}$ has been detected in O-rich binary post-AGB stars (Gielen et al., 2011), contrary to what is required by the bottom-up route ${ }^{4}$.

In Table 2 we compare the effective temperatures of the central stars and the other emission features of $\mathrm{C}_{60}$ objects. They all have effective temperatures of $<35,000 \mathrm{~K}$, suggesting that fullerenes cannot form or survive in strong UV-radiation fields. This situation is similar to that of the unidentified $21 \mu \mathrm{m}$ feature that has been discovered in a small number of PPNs (Kwok et al., 1989). Most of the sources exhibit AIBs arising from $\mathrm{C}-\mathrm{H}$ modes, consistent with the experimental results of hydrocarbon combustion. Not all of them show the $30 \mu \mathrm{m}$ feature and the $15-20 \mu \mathrm{m}$ plateau. It is intriguing that the plateau emission at 6$10 \mu \mathrm{m}$ and $10-14 \mu \mathrm{m}$ is revealed in all the sources, indicating that their carriers are likely to be related to fullerene formation. Kwok and Zhang (2011) presented that AIBs and plateau emission can be uniformly attributed to stretching and bending modes of mixed aromatic-aliphatic organic nanoparticles (MAONs), and during stellar evolution, aliphatic chains can be processed into aromatic rings. García-Hernández et al. (2010) and Micelotta et al. (2012) proposed that fullerenes can be formed from HAC grains and "arophatic" clusters, both of which have a structure similar to that of MAONs. Thus, we infer that circumstellar fullerenes are produced by MAONs following the scheme suggested by Micelotta et al. (2012). In this scenario, protofullerenes, as proposed by Duley and $\mathrm{Hu}$ (2012), are first formed through dehydrogenation of MAONs accompanied by introduction of pentagons following the IPR and a pathway of minimizing the number of dangling bonds, and later on are processed into vibrational-excited closed cages by reducing the number of dangling bonds to zero. The fullerenic cages can be transformed into the most stable isomers by means of a series of Stone-Wales rearrangements. Finally, the fullerenic cages are shrink to smaller cages like $\mathrm{C}_{60}$ by releasing excess energy. The dehydrogenation may be induced by either UV photons or shocks, but the latter seems to be favored at least for a few $\mathrm{C}_{60}$ sources whose central stars have relatively low effective temperatures $(<7000 \mathrm{~K}$; Table 2). The percentage of carbon in circumstellar $\mathrm{C}_{60}$ is estimated to be less than $0.3 \%$ (Zhang and Kwok, 2011; García-Hernández et al., 2012a). Since the carriers of AIBs presumably lock up $6 \%$ of the cosmic carbon (Cerrigone et al., 2009), we infer that only small amounts of MAONs can be transformed into $\mathrm{C}_{60}$ even under the most favorable conditions.

Because MAONs have a 3D structure, the formation process of fullerenic cages can take place in a layered structure. This provides a possible route for the formation of buckyonions, which have been suggested as the carrier of the $217 \mathrm{~nm}$ interstellar absorption feature.

The $\mathrm{C}_{70} / \mathrm{C}_{60}$ abundance ratio has important implications in understanding the formation route of fullerenes. The $\mathrm{C}_{70} / \mathrm{C}_{60}$ ratio of PNs ranges from 0.02 to 0.21 (GarcíaHernández et al., 2012a), comparable to the values of geological fullerenes (0.08 in the Allende meteorite, Becker

\footnotetext{
${ }^{4}$ The detection of $\mathrm{C}_{60}$ in O-rich sources is rare. It is unclear whether $\mathrm{C}_{60}$ in these sources was produced through a different mechanism with those in C-rich objects.
}

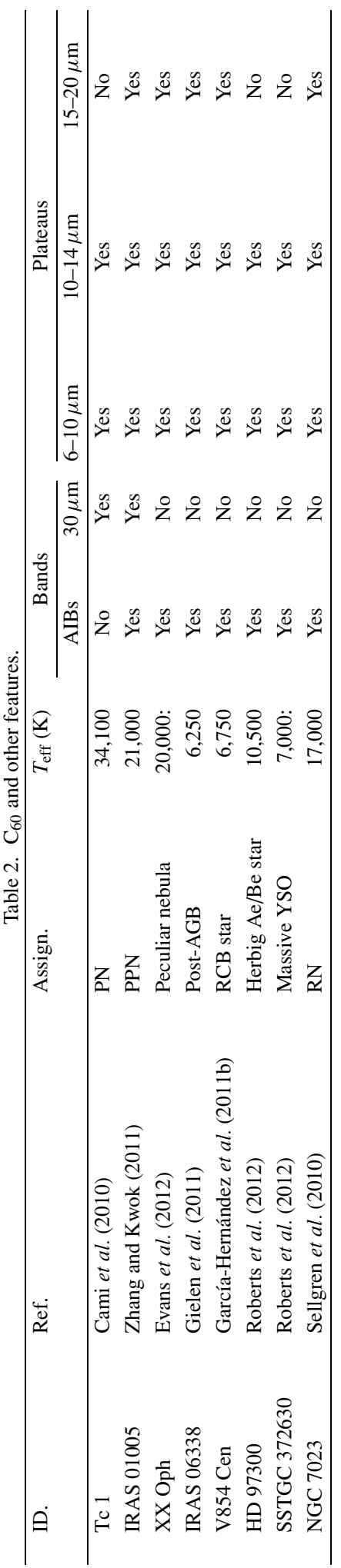


et al., 1994; 0.21-0.36 in terrestrial clays, Heymann et al., 1996). In laboratory, the $C_{70} / C_{60}$ ratio is highly variable. The hydrocarbon combustion process yields a $\mathrm{C}_{70} / \mathrm{C}_{60}$ ratio ranging from 0.26 to 0.57 (Howard et al., 1991), which tends to increase with increasing pressure. This ratio is much larger than that obtained through graphite evaporation methods (0.02-0.18, e.g. Ajie et al., 1990). One cannot simply argue that the low $\mathrm{C}_{70} / \mathrm{C}_{60}$ ratio in $\mathrm{PNs}$ seems not to favor the top-down scenario, because the circumstellar envelopes have a significantly lower pressure than laboratories and the fullerene formation in combustion experiments is not completely understood. Moreover, this range of $\mathrm{C}_{70} / \mathrm{C}_{60}$ ratio is very uncertain since $\mathrm{C}_{70}$ has been measured only in a very few sources. The abundance of generated higher fullerenes beyond $\mathrm{C}_{70}$ is relatively low, which cannot be theoretically explained. In addition, we cannot rule out the possibility that the $\mathrm{C}_{70} / \mathrm{C}_{60}$ ratio can be modified by chemical processes in circumstellar envelopes. Circumstellar envelopes around evolved stars, therefore, provide an unique laboratory to investigate fullerene formation and processes under low pressure environments.

\section{Derivatives of $\mathbf{C}_{60}$ \\ 5.1 A possible detection of fullerane in IRAS $01005+7910$}

The pioneer work of Webster (1992) suggested that the wavelength of the aliphatic $\mathrm{C}-\mathrm{H}$ stretching vibration may shifts toward longer wavelengths from $3.4 \mu \mathrm{m}$ due to the absence of hydrogen atoms on neighboring carbon atoms. Each carbon atom of $\mathrm{C}_{60} \mathrm{H}_{m}$ has three neighboring carbon atoms, results in three possibilities of the absence of hydrogen atoms. Therefore, $\mathrm{C}_{60} \mathrm{H}_{m}$ is expected to exhibit three peaks in the wavelength range longer than $3.4 \mu \mathrm{m}$. The experimental spectra of $\mathrm{C}_{60} \mathrm{H}_{18}$ (Iglesias-Groth et al., 2012) do exhibit three peaks in the wavelength range from 3.4$3.6 \mu \mathrm{m}$. Iglesias-Groth et al. (2012) proposed that because the $\mathrm{C}-\mathrm{H}$ stretching band of $\mathrm{C}_{60} \mathrm{H}_{m}$ is intense and can be easily distinguished from other features, it can serve as an indicator to search for these molecules in astrophysical environments. This band, however, is out of the wavelength range accessible to Spitzer/IRS.

Previously, we have detected $\mathrm{C}_{60}$ and AIBs in IRAS 01005+7910 (Zhang and Kwok, 2011). If $\mathrm{C}_{60}$ and hydrogen are located in the same region, fulleranes are very likely to be present in this object. As a PPN, IRAS $01005+7910$ does not emit atomic lines, and thus provides a good platform to search for fulleranes. The ISO spectrum of IRAS 01005+7910 has been described by Hrivnak et al. (2000). Figure 6 displays the continuum-subtracted ISO spectrum of IRAS $01005+7910$ in the $\mathrm{C}-\mathrm{H}$ stretching region. The $s p^{2} \mathrm{C}-\mathrm{H}$ stretch at $3.3 \mu \mathrm{m}$ has been detected by Hrivnak $e t$ al. (2000). The weak peak at $3.42 \mu \mathrm{m}$ might be due to the asymmetric stretch of $-\mathrm{CH}_{2}$ - groups or just an artifact. The three peaks, presumably ascribed to fulleranes by Webster (1992), are clearly visible at $3.48,3.51$, and $3.58 \mu \mathrm{m}$, and have fluxes comparable to the $3.3 \mu \mathrm{m}$ feature. Through a multi-Gaussian fitting, we estimate the fluxes of the four features, corresponding to $0,1,2$, and 3 non-H-bonded neighboring carbon atoms (referred as $\mathrm{R}_{0}, \mathrm{R}_{1}, \mathrm{R}_{2}$, and $\mathrm{R}_{3}$ hereafter), to be $1.64 \times 10^{-15}, 2.69 \times 10^{-15}, 1.78 \times 10^{-15}$, and $1.61 \times 10^{-15} \mathrm{~W} \mathrm{~m}^{-2}$, respectively. Note that the $\mathrm{R}_{3}$ feature might be blended with an unknown feature (Fig. 6).

Assuming that the $\mathrm{C}_{60}$ emission and the $\mathrm{C}-\mathrm{H}$ stretching emission of $\mathrm{C}_{60} \mathrm{H}_{m}$ origin from UV-excitation, the fractions of carbon locked up in fullerenes and fulleranes $\left(f_{\mathrm{C}}^{\mathrm{C}_{60}}\right.$ and $f_{\mathrm{C}}^{\mathrm{C}_{60} \mathrm{H}_{m}}$ ) can be estimated using the method described by Berné and Tielens (2012). Based on the fluxes of $\mathrm{C}_{60}$ bands given by Zhang and Kwok (2011), we find $f_{\mathrm{C}}^{\mathrm{C}_{60}} / f_{\mathrm{C}}^{\mathrm{C}_{60} \mathrm{H}_{m}} \approx$ 1 , suggesting that about 50 percent of fullerenes have been hydrogenated.

Under the assumption that all the features have the same oscillator strength, the relative strengths of $\mathrm{R}_{0}, \mathrm{R}_{1}, \mathrm{R}_{2}$, and $R_{3}$ are equal to the relative probabilities of the four cases $(0$, 1, 2, 3 non-H-bonded neighboring carbon atoms), and thus the relative strengths of the four $\mathrm{C}_{60} \mathrm{H}_{m}$ features can reflect the degree of hydrogenation (the $m$ value). With increasing number of $\mathrm{C}$ atoms bonded with $\mathrm{H}$, the strength of $\mathrm{R}_{3}$ decreases compared to $R_{0}$. Figure 7 depicts the calculated relative strengths as functions of $m$ values. The observed strengths indicate that the $m$ value is very likely to lie within the range from 25-40. This is consistent with the experimental results in which $\mathrm{C}_{60} \mathrm{H}_{36}$ is the dominant product of hydrogenation reaction of $\mathrm{C}_{60}$. Furthermore, experiments show that $\mathrm{C}_{60} \mathrm{H}_{36}$ can be transferred to $\mathrm{C}_{60} \mathrm{H}_{18}$ through thermal annealing. We find that the relative strength of $\mathrm{R}_{3}$ is slightly higher than that expected for $\mathrm{C}_{60} \mathrm{H}_{36}$ (Fig. 7), suggesting that $\mathrm{C}_{60} \mathrm{H}_{18}$ might be also present. On the other hand, the calculations were purely from a mathematical consideration and did not take into account the chemical structure. Therefore, the fraction of $\mathrm{R}_{3}$ relative to $\mathrm{R}_{0}$ might be underestimated due to ignoring the hydrogen-hydrogen repulsion.

IRAS $01005+7910$ does not exhibit the $21 \mu \mathrm{m}$ feature. Many candidate carriers of this feature have been proposed in previous studies (see Zhang et al., 2009, and references therein), among which Justtanont et al. (1996) and GarcíaLario et al. (1999) have attributed it to the mixture of fullerenes with various degree of hydrogenation. The absence of the $21 \mu \mathrm{m}$ feature in IRAS $01005+7910$ and the non-detection of $\mathrm{C}_{60}$ in $21 \mu \mathrm{m}$-detected PPNs do not support this identification.

During the post-AGB phase, the UV radiation field gradually increases with the evolution of the central star, and molecular $\mathrm{H}_{2}$ is more likely to be photodissociated into atomic $\mathrm{H}$. Moreover, shocks created by fast stellar winds tend to dissociate $\mathrm{H}_{2}$ through collisions. This enhances the possibility of hydrogenation of $\mathrm{C}_{60}$. If $\mathrm{C}_{60}$ is highly hydrogenated, the bond-breaking may occur due to large angle strain, and thus destruct this compound. Besides, intense UV light can directly destroy fullerenes. This can account for the fact that fullerenes were never observed in evolved PNs.

\section{$5.2 \mathrm{C}_{60}^{+}$and $\mathrm{C}_{60}^{-}$in Tc 1}

$\mathrm{C}_{60}$ has an ionization potential of $7.6 \mathrm{eV}$, and thus is possibly present as the cation $\mathrm{C}_{60}^{+}$in circumstellar environments. Ionized $\mathrm{C}_{60}$ may contribute to the diffuse interstellar bands and initiate intriguing chemical reactions (e.g. Foing and Ehrenfreund, 1994; Moutou et al., 1999; Petrie and Bohme, 2000; Leidlmair et al., 2011). $\mathrm{C}_{60}^{-}$is generally thought to be scarce in circumstellar environments 


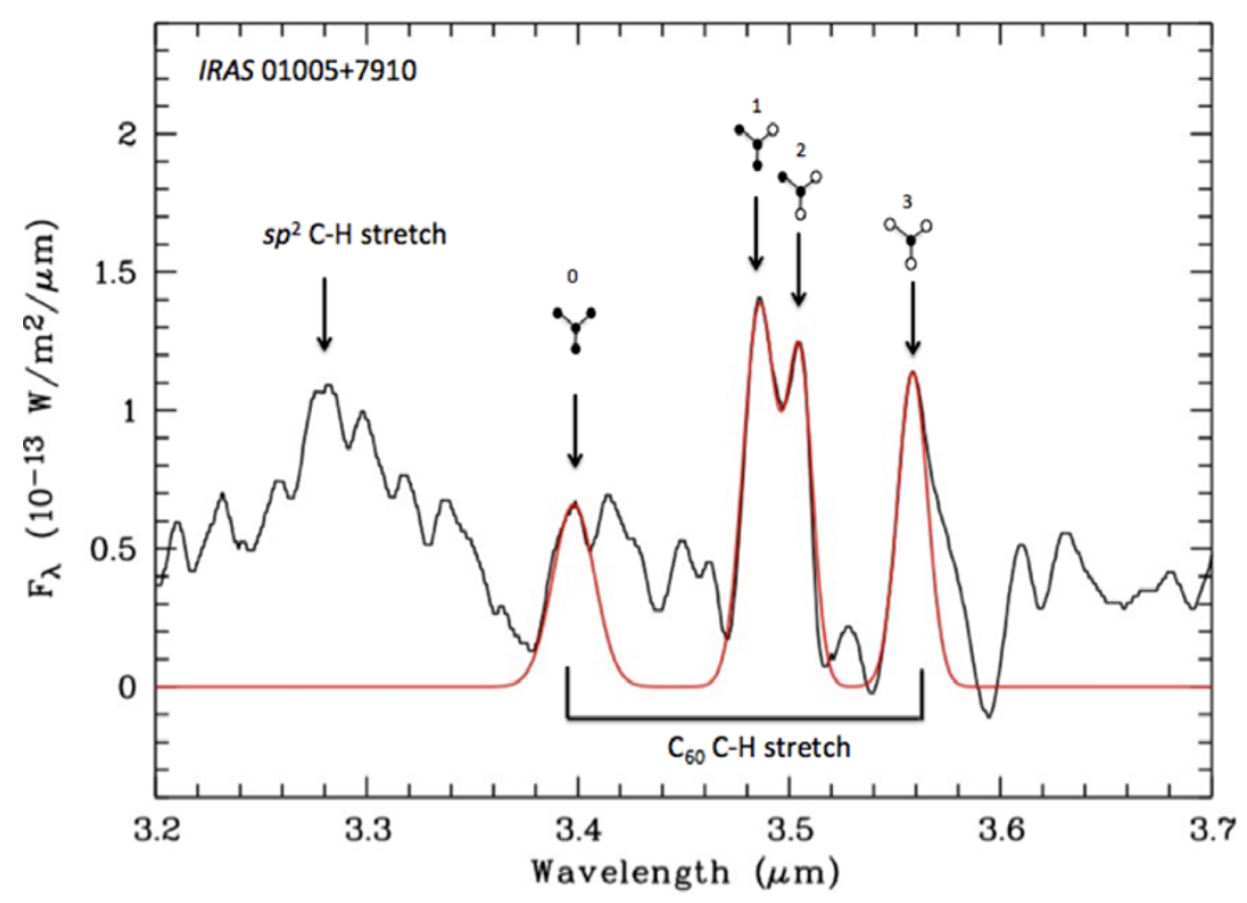

Fig. 6. The ISO spectrum of IRAS $01005+7910$ in the $\mathrm{C}-\mathrm{H}$ stretching region. The peaks for $0,1,2$, and 3 non-H-bonded neighboring carbon atoms are marked. The red line is a multi-Gaussian fitting.

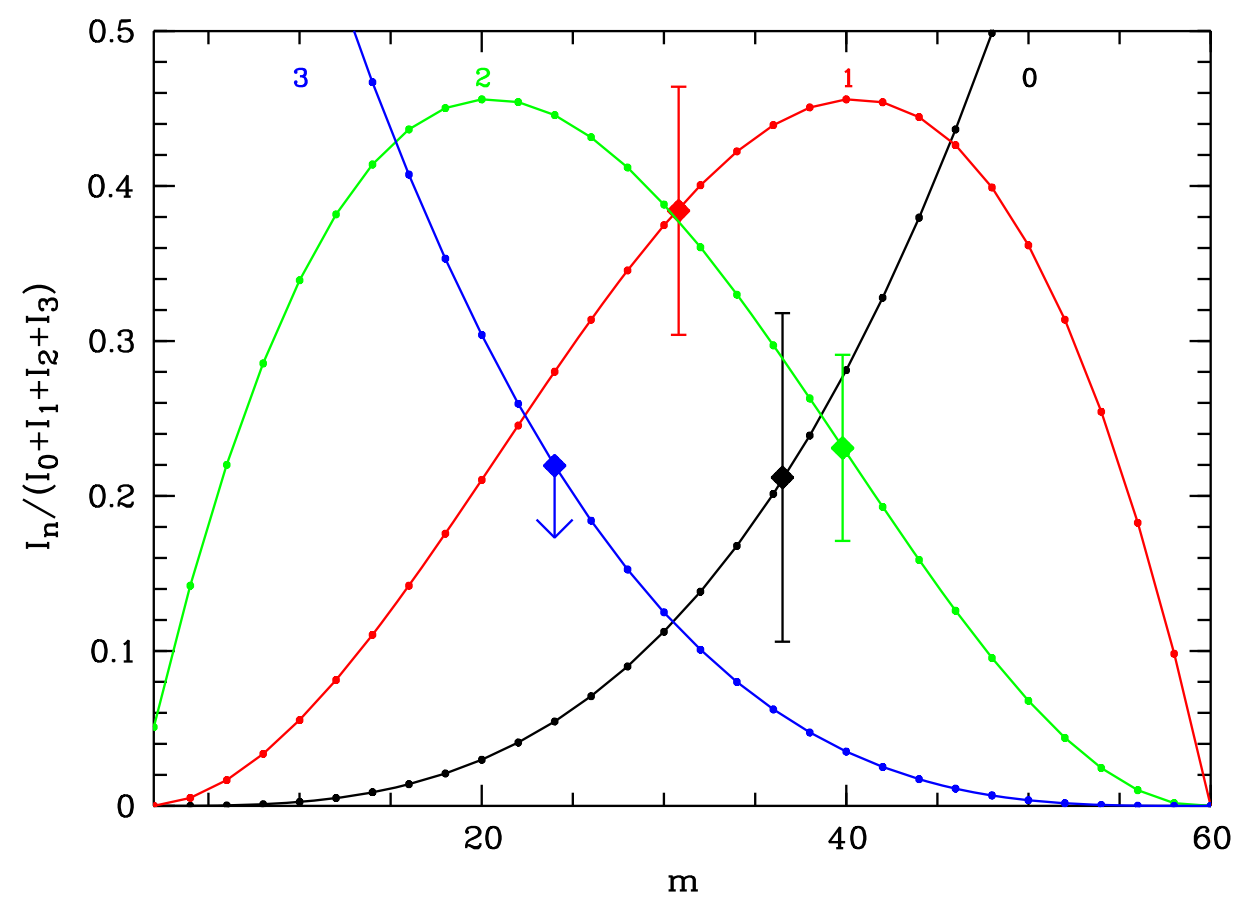

Fig. 7. The calculated probabilities of the four cases $(0$-black, 1 -red, 2 - green, and 3 -blue non-H-bonded neighboring carbon atoms $)$ for a hydrogen bonded carbon atom of $\mathrm{C}_{60} \mathrm{H}_{m}$ vs. the $m$ values. The observed fractional strengths of the four corresponding features are overplotted with lozenges.

in that electron attachment to $\mathrm{C}_{60}$ is prohibited due to its high activation barrier $(0.26 \mathrm{eV})$. However, there are alternative routes generating $\mathrm{C}_{60}^{-}$(Petrie and Bohme, 2000). Recently, Berné et al. (2013) detected $\mathrm{C}_{60}^{+}$IR emission bands in NGC 7023. $\mathrm{C}_{60}^{-}$have never been detected in space so far.

Based on an experiment in $5 \mathrm{~K}$ neon matrices, Fulara et al. (1993) obtained the IR spectrum of $\mathrm{C}_{60}^{+}$and $\mathrm{C}_{60}^{-}$, which reveals the vibrational features at 7.11 and $7.51 \mu \mathrm{m}$ from $\mathrm{C}_{60}^{+}$, and those at 7.22 and $8.32 \mu \mathrm{m}$ from $\mathrm{C}_{60}^{-}$. Figure 8 shows the spectrum of Tc 1 , where the wavelengths of $\mathrm{C}_{60}^{+}$ and $\mathrm{C}_{60}^{-}$bands are indicated. It is clear that there is no detectable $\mathrm{C}_{60}^{-}$emission, and thus the content of circumstellar $\mathrm{C}_{60}^{-}$is safely negligible. However, the presence of $\mathrm{C}_{60}^{+}$ cannot be ruled out. Although the strong forbidden line and $\mathrm{C}_{60}$ band hamper the detection of the $7.11 \mu \mathrm{m}$ band, there is a blended feature on the red side of the $\mathrm{H}$ I $(\operatorname{Pf} \alpha)$ line at $7.46 \mu \mathrm{m}$, which may be partially contributed by the $\mathrm{C}_{60}^{+} 7.51 \mu \mathrm{m}$ band. After subtracting the continuum and 


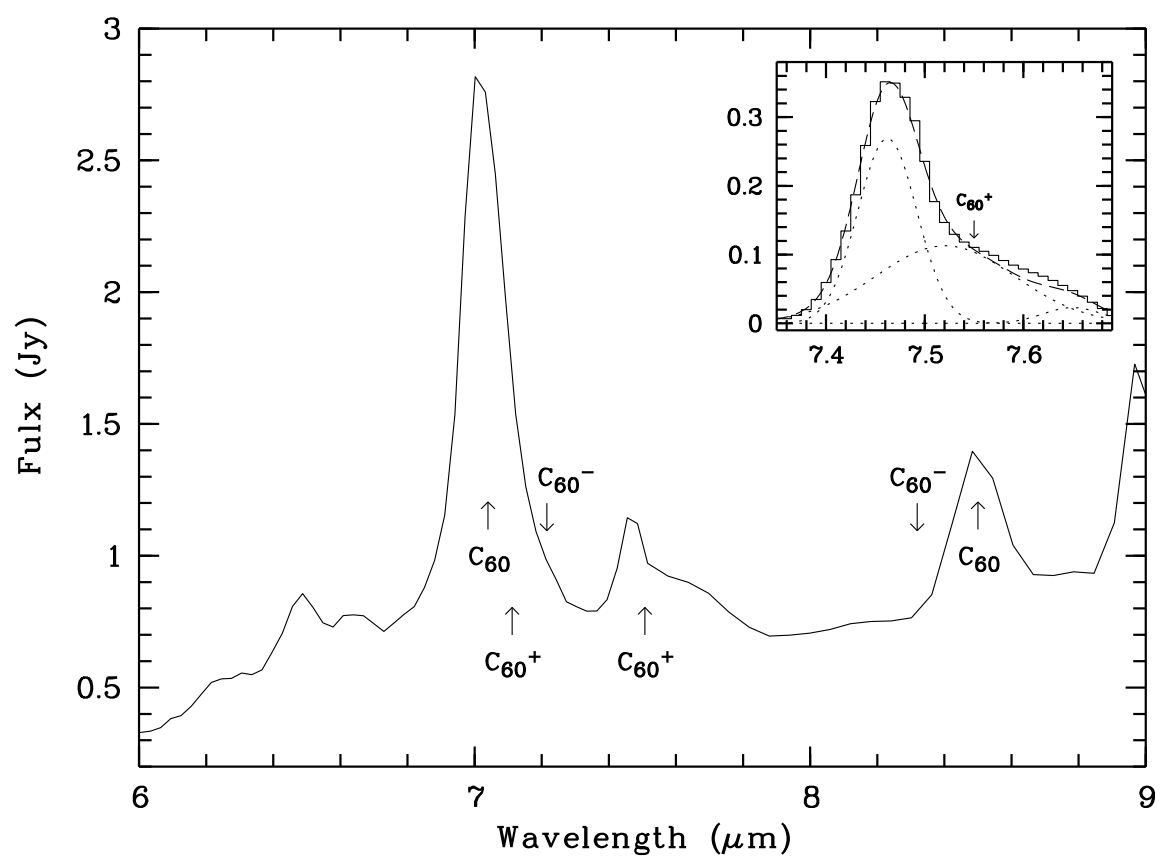

Fig. 8. The Spitzer spectrum of Tc 1 at $6-9 \mu \mathrm{m}$. The experimental wavelengths of $\mathrm{C}_{60}, \mathrm{C}_{60}^{+}$, and $\mathrm{C}_{60}^{-}$are marked. Note that there is a strong [Ar II] forbidden line at $7.0 \mu \mathrm{m}$. The decomposition of the $\mathrm{C}_{60}^{+} 7.51 \mu \mathrm{m}$ feature is shown in the insert where the solid and dashed curves respectively represent the observed and fitted spectra, and the dotted curves denote the individual components.

6-10 $\mu \mathrm{m}$ plateau emission, we decompose this feature, as shown in the insert of Fig. 8. A broaden $7.51 \mu \mathrm{m}$ band with a FWHM of $0.1 \mu \mathrm{m}$ and two narrow atomic lines that are interpreted as $\mathrm{H}$ I and [Ne $\mathrm{VI}$ ] lines can reasonably fit the observations. According to the fitting, the flux of the $\mathrm{C}_{60}^{+}$ $7.51 \mu \mathrm{m}$ band is estimated to be about $1.0 \times 10^{-15} \mathrm{~W} \mathrm{~m}^{-2}$.

The abundance of $\mathrm{C}_{60}^{+}$can be determined using the formulae given by Moutou et al. (1999). For the calculations (see equation 4 of Moutou et al., 1999), the C/H abundance ratio was taken from Pottasch et al. (2011), and the $7.11 \mu \mathrm{m} / 7.51 \mu \mathrm{m}$ cross-section ratio was assumed to be 2.2. Based on a blackbody fitting of the Spitzer spectrum of Tc 1, we estimate that the total IR emission from dust is $(1.5-3.0) \times 10^{-12} \mathrm{~W} \mathrm{~m}^{-2}$. As a result, we obtained that about $0.12 \%-0.23 \%$ carbon is locked up in $\mathrm{C}_{60}^{+}$in Tc 1 if the detection is real. This is comparable to the upper limit of $0.26 \%$ in NGC 7023 (Moutou et al., 1999), and the value of $0.3 \%-0.9 \%$ in the ISM that was estimated by Foing and Ehrenfreund (1994) from two near-IR diffuse bands.

However, as noted recently by Berné et al. (2013), a new spectroscopic measurement performed by Kern et al. (2012) suggests that the $7.51 \mu \mathrm{m}$ band might be due to $\mathrm{C}_{60}^{-}$rather than $\mathrm{C}_{60}^{+}$. Thus the above discussion is only tentative.

\section{Conclusions}

$\mathrm{C}_{60}$ and higher fullerenes have been detected in circumstellar and geological environments. This strengthens the idea that a variety of carbon-based compounds, including fullerenes, can be efficiently produced by stars, be ejected into the ISM, reach the early Solar System, and be partially brought to Earth by comets and asteroids (Kwok, 2011). The study of $\mathrm{C}_{60}$ and its derivatives in circumstellar envelopes can help to understand the chemical evolution of galaxies. However, it is unclear how circumstellar
$\mathrm{C}_{60}$ is formed and excited. In this paper, we investigate the relations between the emission properties of $\mathrm{C}_{60}$-detected sources, as well as between the observations and existing experimental results. Through a comparison of wavelengths and fluxes of $\mathrm{C}_{60}$ bands, we conclude that the UV-excitation of $\mathrm{C}_{60}$ in cluster state may account for the observations, although other excitation schemes, such as the release of chemical energy, remain possible. We would like to emphasize that the Einstein A-values and flux measurements are too uncertain to allow definite conclusions. We also propose that $\mathrm{C}_{60}$ is one of the products of the dehydrogenation of MAONs through a scenario presented by Micelotta et al. (2012). The fullerene formation thus reflects a transformation from $s p^{3}$ to $s p^{2}$ hybridization in MAONs. We have tentatively detected hydrogenated $\mathrm{C}_{60}$ in IRAS $01005+7910$ and $\mathrm{C}_{60}^{+}$in $\mathrm{Tc} 1$. The presence of $\mathrm{C}_{60}$ derivatives has the implications that fullerenes have been UV photochemically processed during the post-AGB evolution and the $\mathrm{C}_{70} / \mathrm{C}_{60}$ ratio can be significantly modified. A combination of further experiments and observations are required to obtain complete picture of fullerene formation and processes in circumstellar environments.

Acknowledgments. YZ would like to thank the SOC of the 5th meeting on Cosmic Dust for the invitation to give this talk at Center for Planetary Science, c/o Integrated Research Center of Kobe University. We also thank two anonymous referees for helpful comments. Financial support for this work was provided by the Research Grants Council of the Hong Kong under grants HKU7073/11P.

\section{References}

Adams, G. B., J. B. Page, O. F. Sankey, and M. O'Keeffe, Polymerized $\mathrm{C}_{60}$ studied by first-principles molecular dynamics, Phys. Rev. B, 50, 17471-17479, 1994.

Ajie, H. et al., Characterization of the soluble all-carbon molecules $\mathrm{C}_{60}$ 
and $\mathrm{C}_{70}$, J. Phys. Chem., 94, 8630-8633, 1990.

Allamandola, L. J., A. G. G. M. Tielens, and J. R. Barker, Interstellar polycyclic aromatic hydrocarbons-The infrared emission bands, the excitation/emission mechanism, and the astrophysical implications, Astrophys. J. Suppl., 71, 733-775, 1989.

Becker, L. and J. L. Bada, Fullerenes in Allende meteorite, Nature, 372, 507-507, 1994.

Becker, L., J. L. Bada, R. E. Winans, J. E. Hunt, T. E. Bunch, and B. M. French, Fullerenes in the 1.85-billion-year-old Sudbury impact structure, Science, 265, 642-645, 1994.

Bernard-Salas, J., J. Cami, E. Peeters, A. P. Jones, E. R. Micelotta, and M. A. T. Groenewegen, On the excitation and formation of circumstellar fullerenes, Astrophys. J., 757, 41, 2012.

Berné, O. and A. G. G. M. Tielens, Formation of buckminsterfullerene $\left(\mathrm{C}_{60}\right)$ in interstellar space, PNAS, 109, 401-406, 2012.

Berné, O., G. Mulas, and C. Joblin, Interstellar $\mathrm{C}_{60}{ }^{+}$, Astron. Astrophys., 550, L4, 2013.

Bertsch, G. F., A. Smith, and K. Yabana, Optical properties of $\mathrm{C}_{60}$ vibrations, Phys. Rev. B, 52, 7876-7878, 1995.

Bochvar, D. A. and E. G. Galpern, Hypothetical systems: carbododecahedron, s-icosahedron, and carbo-s-icosahedron, Dokl. Acad. Nauk SSSR, 209, 610-612, 1973 (in Russian).

Boersma, C., R. H. Rubin, and L. J. Allamandola, Spatial analysis of the polycyclic aromatic hydrocarbon features southeast of the Orion Bar, Astrophys. J., 753, 168, 2012.

Buseck, P. R., Geological fullerenes: review and analysis, Earth Planet. Sci. Lett., 203, 781-792, 2002.

Buseck, P. R., S. J. Tsipursky, and R. Hettich, Fullerenes from the geological environment, Science, 257, 215-217, 1992.

Cami, J., J. Bernard-Salas, E. Peeters, and S. E. Malek, Detection of $\mathrm{C}_{60}$ and $\mathrm{C}_{60}$ in a young planetary nebula, Science, 329, 1180-1182, 2010.

Cataldo, F. and S. Iglesias-Groth, On the action of UV photons on hydrogenated fulleranes $\mathrm{C}_{60} \mathrm{H}_{36}$ and $\mathrm{C}_{60} \mathrm{D}_{36}$, Mon. Not. R. Astron. Soc., 400, 291-298, 2009.

Chase, B., N. Herron, and E. Holler, Vibrational spectroscopy of fullerenes $\left(\mathrm{C}_{60}\right.$ and $\left.\mathrm{C}_{70}\right)$ temperature dependant studies, J. Phys. Chem., 96, 42624266, 1992.

Cerrigone, L., J. L. Hora, G. Umana, and C. Trigilio, Spitzer Detection of polycyclic aromatic hydrocarbons and silicate features in post-AGB stars and young planetary nebulae, Astrophys. J., 703, 585, 2009.

Choi, C. H., M. Kertesz, and L. Mihaly, Vibrational assignment of all 46 fundamentals of $\mathrm{C}_{60}$ and $\mathrm{C}_{60}^{6-}$ : Scaled quantum mechanical results performed in redundant internal coordinates and compared to experiments, J. Phys. Chem. A, 104, 102-112, 2000.

Clayton, G. C., D. M. Kelly, J. H. Lacy, I. R. Little-Marenin, P. A. Feldman, and P. F. Bernath, A mid-infrared search for $\mathrm{C}_{60}$ in R Coronae Borealis stars and IRC+10216, Astron. J., 109, 2096, 1995.

Clayton, G. C. et al., The dust properties of two hot R Coronae Borealis stars and a Wolf-Rayet central star of a planetary nebula: In search of a possible link, Astron. J., 142, 54, 2011.

de Heer, W. A. and D. Ugarte, Carbon onions produced by heat treatment of carbon soot and their relation to the $217.5 \mathrm{~nm}$ interstellar absorption feature, Chem. Phys. Lett., 207, 480-486, 1993.

de Vries, M. S. et al., A search for $\mathrm{C}_{60}$ in carbonaceous chondrites, Geochim. Cosmochim. Acta, 57, 933-938, 1993.

Duley, A. A. and A. Hu, Fullerenes and proto-fullerenes in interstellar carbon dust, Astrophys. J., 745, L11, 2012.

Duley, A. A. and D. A. Williams, Excitation of the aromatic infrared emission bands: Chemical energy in hydrogenated amorphous carbon particles?, Astrophys. J., 737, L44, 2011.

Ebbesen, T. W. et al., Origins of fullerenes in rocks, Science, 268, 1634$1635,1995$.

Esfarjani, K., Y. Hashi, J. Onoe, K. Takeuchi, and Y. Kawazoe, Vibrational modes and IR analysis of neutral photopolymerized $\mathrm{C}_{60}$ dimers, Phys. Rev. B, 57, 223-229, 1998.

Evans, A. et al., Solid-phase $\mathrm{C}_{60}$ in the peculiar binary $\mathrm{XX}$ Oph?, Mon. Not. R. Astron. Soc., 421, L92, 2012.

Foing, B. H. and P. Ehrenfreund, Detection of two interstellar absorption bands coincident with spectral features of $\mathrm{C}_{60}^{+}$, Nature, 369, 296-298, 1994.

Frum, C. I., R. Engelman, H. G. Hedderich, P. F. Bernath, L. D. Lamb, and D. R. Huffman, The infrared emission spectrum of gas-phase $\mathrm{C}_{60}^{+}$ (buckmisterfullerene), Chem. Phys. Lett., 176, 504-508, 1991.

$\mathrm{Fu}$, K.-J. et al., Giant vibrational resonances in $\mathrm{A}_{6} \mathrm{C}_{60}$ compounds, Phys. Rev. B, 46, 1937-1940, 1992.

Fulara, J., M. Jakobi, and J. P. Maier, Electronic and infrared spectra of
$\mathrm{C}_{60}^{+}$and $\mathrm{C}_{60}^{-}$in neon and argon matrices, Chem. Phys. Lett., 21, 227$234,1993$.

García-Hernández, D. A. and J. J. Díaz-Luis, Diffuse interstellar bands in fullerene planetary nebulae: The fullerenes-diffuse interstellar bands connection, Astron. Astrophys., 550, L6, 2013.

García-Hernández, D. A. et al., Formation of fullerenes in H-containing planetary nebulae, Astrophys. J., 724, L39, 2010.

García-Hernández, D. A. et al., The formation of fullerenes: Clues from new $\mathrm{C}_{60}, \mathrm{C}_{60}$, and (possible) planar $\mathrm{C}_{60}$ detections in Magellanic Cloud planetary nebulae, Astrophys. J., 737, L30, 2011a.

García-Hernández, D. A., R. N. Kameswara, and D. L. Lambert, Are $\mathrm{C}_{60}$ molecules detectable in circumstellar shells of $\mathrm{R}$ Coronae Borealis stars?, Astrophys. J., 729, 126, 2011 b.

García-Hernández, D. A. et al., Infrared study of fullerene planetary nebulae, Astrophys. J., 760, 197, 2012a.

García-Hernández, D. A., R. N. Kameswara, and D. L. Lambert, Highresolution optical spectroscopy of DY Cen: Diffuse interstellar bands in a proto-fullerene circumstellar environment?, Astrophys. J., 759, L21, 2012 b.

García-Lario, P., A. Manchado, A. Ulla, and M. Manteiga, Infrared space observatory observations of IRAS 16594-4656: A new Proto-planetary nebula with a strong 21 micron dust feature, Astrophys. J., 513, 941, 1999.

Giannozzi, P. and S. Baroni, Vibrational and dielectric properties of $\mathrm{C}_{60}$ from densityfunctional perturbation theory, J. Chem. Phys., 100, 8537, 1994.

Gielen, C., J. Cami, J. Bouwman, E. Peeters, and M. Min, Carbonaceous molecules in the oxygen-rich circumstellar environment of binary postAGB stars. $\mathrm{C}_{60}$ fullerenes and polycyclic aromatic hydrocarbons, Astron. Astrophys., 536, 54, 2011.

Goeres, A. and E. Sedlmayr, The envelopes of R Coronae Borealis stars. I-A physical model of the decline events due to dust formation, Astron. Astrophys., 265, 216-236, 1992.

Hara, T., J. Onoe, and K. Takeuchi, In Situ High-Resolution FT-IR Study of the Glass Transition in a $\mathrm{C}_{60}$ Film, J. Phys. Chem. B, 101, 9532-9536, 1997.

Herbig, G. H., The search for interstellar $\mathrm{C}_{60}$, Astrophys. J., 542, 334-343, 2000.

Heymann, D., Astrophys. J., 489, 111, 1997.

Heymann, D. et al., Fullerenes of possible wildfire origin in CretaceousTertiary boundary sediments, Geol. Soc. Am. Spec. Pap., 307, 453-464, 1996.

Houck, J. R. et al., The Infrared Spectrograph (IRS) on the Spitzer Space Telescope, Astrophys. J. Suppl., 154, 18-24, 2004.

Howard, J. B., J. T. McKinnon, Y. Makarovsky, A. L. Lafleur, and M. E. Johnson, Fullerenes $\mathrm{C}_{60}$ and $\mathrm{C}_{70}$ in flames, Nature, 352, 139-141, 1991.

Hrivnak, B. J., K. Volk, and S. Kwok, 2-45 micron infrared spectroscopy of carbon-rich proto-planetary nebulae, Astrophys. J., 535, 275-292, 2000.

Iglesias-Groth, S., Fullerenes and buckyonions in the interstellar medium, Astrophys. J., 608, L37-L40, 2004.

Iglesias-Groth, S., A. Ruiz, J. Bretón, and J. M. Gómez Llorente, Photoabsorption spectra of icosahedral fullerenes: A semiempirical approach, $J$. Chem. Phys., 116, 10648, 2002.

Iglesias-Groth, S., F. Cataldo, and A. Manchado, Infrared spectroscopy and integrated molar absorptivity of $\mathrm{C}_{60}$ and $\mathrm{C}_{70}$ fullerenes at extreme temperatures, Mon. Not. R. Astron. Soc., 413, 213-222, 2011.

Iglesias-Groth, S., D. A. García-Hernández, F. Cataldo, and A. Manchado, Infrared spectroscopy of hydrogenated fullerenes (fulleranes) at extreme temperatures, Mon. Not. R. Astron. Soc., 423, 2868-2878, 2012.

Jäger, C., F. Huisken, H. Mutschke, I. L. Jansa, and T. Henning, Formation of Polycyclic aromatic hydrocarbons and carbonaceous solids in gasphase condensation experiments, Astrophys. J., 696, 706-712, 2009.

Justtanont, K., M. J. Barlow, C. J. Skinner, P. F. Roche, D. K. Aitken, and C. H. Smith, Mid-infrared spectroscopy of carbon-rich post-AGB objects and detection of the PAH molecule chrysene, Astron. Astrophys., 309, 612-628, 1996.

Kern, B., D. Strelnikov, P. Weis, A. Böttcher, and M. M. Kappes, $\mathrm{C}_{60}^{+}$and $\mathrm{C}_{60}^{-}$in neon and argon matrices, in 67th International Symposium on Molecular Spectroscopy, 2012.

Kessler, M. F. et al., The Infrared Space Observatory (ISO) mission, Astron. Astrophys., 315, L27-L31, 1996.

Krätschmer, W., The infrared and ultraviolet absorption spectra of laboratory-produced carbon dust: Evidence for the presence of the $\mathrm{C}_{60}$ molecule, K. Fostiropoulos, and D. R. Huffman, Chem. Phys. Lett., 170, 167-170, 1990a.

Krätschmer, W., L. D. Lamb, K. Fostiropoulos, and D. R. Huffman, C 60 , a 
new form of carbon, Nature, 347, 354-358, 1990b.

Kroto, H. W., J. R. Heath, S. C. Obrien, R. F. Curl, and R. E. Smalley, C60: buckminsterfullerene, Nature, 318, 162-163, 1985.

Kwok, S., Amorphous organic solids as a component of interstellar dust, Earth Planets and Space, 63, 1021-1026, 2011.

Kwok, S. and Y. Zhang, Mixed aromatic-aliphatic organic nanoparticles as carriers of unidentified infrared emission features, Nature, 479, 80-83, 2011.

Kwok, S., K. Volk, and B. J. Hrivnak, A 21 micron emission feature in four proto-planetary nebulae, Astrophys. J., 345, L51-L54, 1989.

Kwok, S., S. Volk, and B. J. Hrivnak, Chemical evolution of carbonaceous materials in the last stages of stellar evolution, Astron. Astrophys., 350, L35-L38, 1999.

Léger, A., L. D'Hendecourt, L. Verstraete, and W. Schmidt, Remarkable candidates for the carrier of the diffuse interstellar bands-C60(+) and other polyhedral carbon ions, Astron. Astrophys., 203, L145-L148, 1988.

Leidlmair, C. et al., On the possible presence of weakly bound fullerene$\mathrm{H}_{2}$ complexes in the interstellar medium, Astrophys. J., 738, L4, 2011.

Li, A., J.-H. Chen, M.-P. Li, Q.-J. Shi, and Y.-J. Wang, On buckyonions as an interstellar grain component, Mon. Not. R. Astron. Soc., 390, 39-42, 2008.

Martin, M. C., D. Koller, and L. Mihaly, In situ infrared transmission study of Rb- and K-doped fullerenes, Phys. Rev. B, 47, 14607-14610, 1993.

Micelotta, E. R., A. P. Jones, J. Cami, E. Peeters, J. Bernard-Salas, and G. Fanchini, The formation of cosmic fullerenes from arophatic clusters, Astrophys. J., 761, 35, 2012.

Moutou, C., K. Sellgren, L. Verstraete, and A. Léger, Upper limit on $\mathrm{C}_{60}$ and $\mathrm{C}_{60}(+)$ features in the ISO-SWS spectrum of the reflection nebula NGC 7023, Astron. Astrophys., 347, 949-956, 1999.

Nemes, L. et al., Gas-phase infrared emission spectra of $\mathrm{C}_{60}$ and $\mathrm{C}_{70}$. Temperature-dependent studies, Chem. Phys. Lett., 218, 295-303, 1994.

Onoe, J. and K. Takeuchi, In situ high-resolution infrared spectroscopy of a photopolymerized $\mathrm{C}_{60}$ film, Phys. Rev. B, 54, 6167-6171, 1996.

Osawa, E., Superaromaticity, Kagaku, 25, 854-863, 1970 (in Japanese).

Papoular, R., Excitation of unidentified infrared bands by $\mathrm{H}$ atom impacts, Mon. Not. R. Astron. Soc., 419, 2396-2406, 2012.

Petrie, S. and D. K. Bohme, Laboratory studies of ion/molecule feactions of fullerenes: Chemical derivatization of fullerenes within dense interstellar clouds and circumstellar shells, Astrophys. J., 540, 869-885, 2000.

Pottasch, S. R., R. Surendiranath, and J. Bernard-Salas, Abundances in planetary nebulae: NGC 1535, NGC 6629, He2-108, and Tc1, Astron. Astrophys., 531, A23, 2011.

Roberts, K. R. G., K. T. Smith, and P. J. Sarre, Detection of $\mathrm{C}_{60}$ in embedded young stellar objects, a Herbig Ae/Be star and an unusual postasymptotic giant branch star, Mon. Not. R. Astron. Soc., 421, 32773285, 2012.

Rubin, R. H. et al., Spitzer reveals what is behind Orion's Bar, Mon. Not R. Astron. Soc., 410, 1320-1348, 2011.

Sassara, A., G. Zerza, M. Chergui, and S. Leach, Absorption wavelengths and bandwidths for interstellar searches of $\mathrm{C}_{60}$ in the 2400-4100 $\AA$ region, Astrophys. J. Suppl., 135, 263-273, 2001.

Sellgren, K., M. W. Werner, J. G. Ingalls, J. D. T. Smith, T. M. Carleton, and C. Joblin, $\mathrm{C}_{60}$ in reflection nebulae, Astrophys. J., 722, L54-L57, 2010.

Snow, T. P. and C. G. Seab, A search for interstellar and circumstellar $\mathrm{C}_{60}$, Astron. Astrophys., 213, 291-294, 1989.

Stanton, R. E. and M. D. Newton, Normal vibrational modes of buckminsterfullerene, J. Phys. Chem., 92, 2141-2145, 1988.

Webster, A., Unsaturated fulleranes and the minor features of the unidentified infrared emission near 3 microns, Mon. Not. R. Astron. Soc., 257, 463-470, 1992.

Webster, A., A theory of the diffuse interstellar bands, Mon. Not. R. Astron. Soc., 262, 831-838, 1993.

Werner, M. et al., The Spitzer Space Telescope mission, Astrophys. J. Suppl., 154, 1-9, 2004.

Winkler, R., T. Pichler, and H. Kuzmany, Vibrational analysis of IR reflection-transmission from single crystal $\mathrm{C}_{60}$, Phys. Rev. B, 96, 3945, 1994.

Yagi, H. et al., Photoabsorption cross section of $\mathrm{C}_{60}$ thin films from the visible to vacuum ultraviolet, Carbon, 47, 1152-1157, 2009.

Yasumatsu, H., T. Kondow, H. Kitagawa., K. Tabayashi, and K. Shobatake, Absorption spectrum of $\mathrm{C}_{60}$ in the gas phase: Autoionization via coreexcited Ryberg states, J. Chem. Phys., 104, 899, 1996.

Zhang, K., B.-W. Jiang, and A. Li, On the carriers of the $21 \mu \mathrm{m}$ emission feature in post-asymptotic giant branch stars, Mon. Not. R. Astron. Soc., 396, 1247-1256, 2009.

Zhang, Y. and S. Kwok, Detection of $\mathrm{C}_{60}$ in the protoplanetary nebula IRAS 01005+7910, Astrophys. J., 730, 126, 2011.

Y. Zhang (e-mail: zhangy96@hku.hk) and S. Kwok 